\title{
Skein algebras of the solid torus and symmetric spatial graphs
}

\author{
by
}

\author{
Nafaa Chbili (Tokyo)
}

\begin{abstract}
We use the topological invariant of spatial graphs introduced by S. Yamada to find necessary conditions for a spatial graph to be periodic with a prime period. The proof of the main result is based on computing the Yamada skein algebra of the solid torus and then proving that it injects into the Kauffman bracket skein algebra of the solid torus.
\end{abstract}

1. Introduction. Throughout this paper a graph is a finite one-dimensional CW-complex. We assume that vertices of our graphs have valency greater than or equal to 3 . If $G$ is a graph, we denote by $\mathcal{V}(G)$ the set of vertices and by $\mathcal{E}(G)$ the set of edges of $G$. An embedding of such a graph into the three-sphere is called a spatial graph. Let $p \geq 2$ be an integer. A spatial graph $\widetilde{G}$ is said to be p-periodic if there exists an orientation preserving diffeomorphism $h$ of $\left(S^{3}, \widetilde{G}\right)$ such that $h$ is of order $p$ and the set of fixed points of $h$ is a circle that does not intersect $\widetilde{G}$. If $\widetilde{G}$ is a periodic spatial graph, we will denote the quotient spatial graph by $\widetilde{G}$.

By the positive solution of the Smith conjecture [1], the action defined by $h$ on the three-sphere is topologically conjugate to an orthogonal action. In other words, if we identify the three-sphere with $\mathbb{R}^{3} \cup \infty$, then we may assume that $h$ is the rotation by the angle of $2 \pi / p$ around the standard $z$-axis in the Euclidean space $\mathbb{R}^{3}$. Hence, a $p$-periodic spatial graph may be represented by a diagram in $\mathbb{R}^{2}$ which is invariant under a planar rotation.

Let $\widetilde{G}$ be a $p$-periodic spatial graph. We define the wrapping number of $\widetilde{G}$ to be the minimum number of intersection points of $\widetilde{G}$ with $P$, where $P$ runs over all half-planes bounded by the axis of the rotation [7].

2000 Mathematics Subject Classification: 05C10, 57M25, 57M27.

Key words and phrases: Yamada polynomial, Kauffman bracket skein modules, periodic spatial graphs.

Supported by a fellowship from the Japan Society for the Promotion of Science (JSPS), and by a Grant-in-Aid for JSPS fellows 03020. The author would like to thank the JSPS for its support. 
The periodicity of knots and links has been the subject of extensive literature. Both the new and the classical invariants have been used successfully in this study. In particular, several criteria for periodicity of links have been introduced in terms of invariants of skein type. For instance, using the Jones polynomial (or, equivalently, the Kauffman bracket), Murasugi [8] and Traczyk [11] introduced necessary conditions for a link to be periodic with a prime period. These criteria have been extended to the skein polynomial (HOMFLYPT) by Przytycki [9].

In [13], Yamada introduced a topological invariant of spatial graphs which takes values in $\mathcal{R}=\mathbb{Z}\left[A^{ \pm 1}, d^{-1}\right]$, where $d=-A^{2}-A^{-2}$. This invariant, denoted here by $Y$, can be defined recursively on planar diagrams of spatial graphs. When restricted to knots, this invariant is essentially the Jones polynomial of some cable of the knot.

The goal of this paper is to use the Yamada polynomial to find necessary conditions for a spatial graph to be periodic. In the special cases corresponding to periodic graphs with wrapping number one or two, Marui [7] introduced necessary conditions, in terms of the Yamada polynomial, for a spatial graph to be periodic with wrapping number one or two. The main result in our paper is given by the following theorem, where the congruences hold in the ring $\mathbb{Z}\left[A^{ \pm 1}, d^{-1}\right]$.

TheOREM 1.1. Let $p$ be a prime and $\widetilde{G}$ a spatial graph. If $\widetilde{G}$ is p-periodic, then

(1) $Y(\widetilde{G})(A) \equiv(Y(\widetilde{G})(A))^{p} \bmod p, d^{p}-d$.

(2) $Y(\widetilde{G})(A) \equiv Y(\widetilde{G})\left(A^{-1}\right) \bmod p, A^{2 p}-1$.

Application. Let $P$ be the Peterson graph and $\widetilde{P}$ be the embedding of $P$ given by Figure 1. Marui [7] proved that this spatial graph cannot be 5 -periodic with wrapping number two.

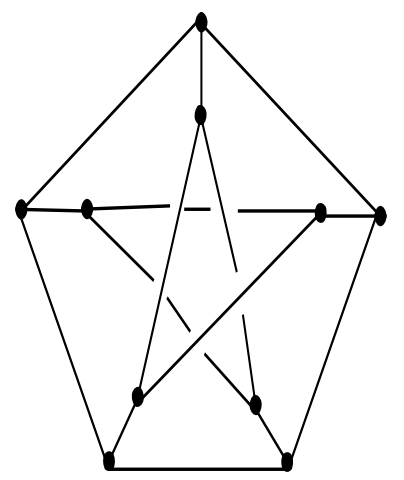

Fig. 1 
If we apply Theorem $1.1(2)$ we can prove that $\widetilde{P}$ is not 5 -periodic with any wrapping number. From the computation in [7], we have the following congruence:

$$
Y(\widetilde{P})(A) \equiv 2\left(4 A^{6}+4+A^{4}+A^{8}\right) \bmod 5, A^{10}-1 .
$$

Obviously this polynomial does not satisfy condition (2) of Theorem 1.1. Hence, the spatial graph $\widetilde{P}$ is not 5 -periodic.

It is worth mentioning here that the finite group $\mathbb{Z} / 5 \mathbb{Z}$ acts freely on the abstract graph $P$.

REMARK. Let $G$ be a graph and let $\widetilde{G}$ be a spatial embedding of $G$ which is $p$-periodic. Then the rotation induces actions of the finite group $\mathbb{Z} / p \mathbb{Z}$ on $\mathcal{E}(G)$ and $\mathcal{V}(G)$. Obviously, the action of $\mathbb{Z} / p \mathbb{Z}$ on $\mathcal{V}(G)$ is free. Moreover, vertices that belong to the same orbit have the same valency. Hence, we can get some restriction on the possible periods for a spatial graph $\widetilde{G}$ from the properties of the original abstract graph; in particular, from the number and valency of vertices. For instance, we can easily see that for all integers $p$ an embedding of a $\theta$-curve cannot be $p$-periodic. Also, an embedding of the complete graph $K_{n}$ cannot be 2-periodic.

2. The Yamada polynomial. A ribbon graph is an oriented compact surface with boundary that retracts by deformation onto a graph. A spatial ribbon graph is an embedding of a ribbon graph into the three-sphere. It is well known that the study of spatial ribbon graphs up to isotopy is equivalent to the study of planar graph diagrams up to extended Reidemeister moves [13].

In [13], S. Yamada introduced an invariant $R$ of regular isotopy of spatial graphs. This invariant takes its values in the ring $\mathbb{Z}\left[A^{ \pm 1}\right]$ and may be defined recursively on diagrams of spatial graphs. A similar invariant of trivalent graphs, with good weight associated with the set of edges, was also introduced by Yamada [14]. This invariant was extended by Yokota [15] using the linear skein theory introduced by Lickorish [6].

In the present paper, we find it more convenient to slightly change the recursive formulas introduced by Yamada. Namely, we define an invariant $Y$ of spatial graphs recursively by the initialization $Y(\emptyset)=1$ and the four relations in Figure 2. Notice that the vertical dots in our figures mean an arbitrary number of edges. It is also worth mentioning that the following identities hold for diagrams which are identical except in a small disk where they look as indicated below.

Throughout this paper, these relations will be referred to as the Yamada skein relations. 


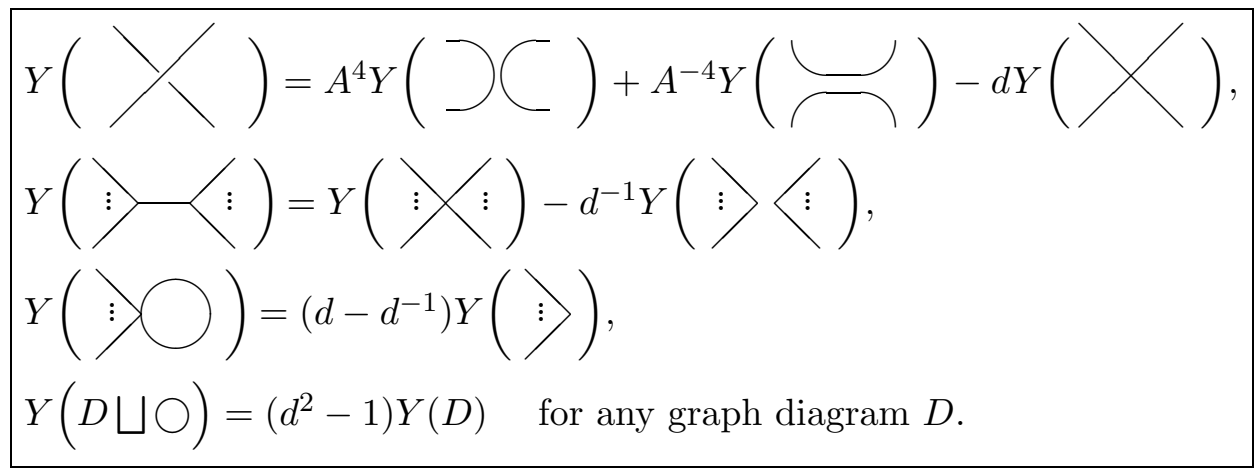

Fig. 2

TheOREM 2.1. $Y$ is an invariant of ribbon spatial graphs.

Proof. Comparing the recursive formulas defining the Yamada polynomial $R$ (see [13, Section 5]) to the formulas in Figure 2, we can easily see that for any spatial graph $\widetilde{G}$ we have

$$
Y(\widetilde{G})(A)=(-d)^{\chi(G)} R(\widetilde{G})\left(A^{4}\right),
$$

where $\chi(G)$ is the Euler characteristic of the graph $G$. Since $R$ is an invariant of ribbon spatial graphs, so is $Y$.

The Kauffman bracket [4] polynomial $\{$,$\} is an invariant of regular iso-$ topy of framed links which can be defined recursively by the relations

$$
\{\bigcirc \cup L\}=d\{L\}, \quad\{L\}=A\left\{L_{0}\right\}+A^{-1}\left\{L_{\infty}\right\},
$$

where $d=-A^{2}-A^{-2}, L, L_{0}$ and $L_{\infty}$ are three links which are identical except in a three-ball where they are as in Figure 3.
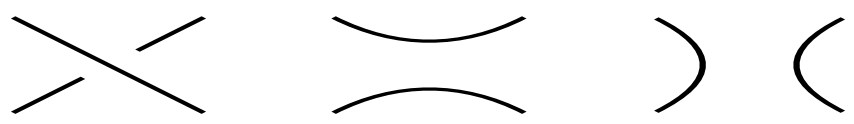

Fig. 3

It is worth mentioning that the Kauffman bracket is a version of the Jones polynomial for unoriented framed links. Murasugi and Traczyk studied the Jones polynomial of periodic links. They provided criteria for periodicity of links in terms of the Jones polynomial. Here, we give corollaries of these criteria using the Kauffman bracket (see also [2]).

Theorem $2.2([8,11])$. Let $p$ be a prime and let $L$ be a p-periodic link. Then

(1) $\{L\} \equiv(\{\bar{L}\})^{p} \bmod p, d^{p}-d$, where $\bar{L}$ is the quotient link.

(2) $\{L\}(A) \equiv\{L\}\left(A^{-1}\right) \bmod p, A^{2 p}-1$. 
3. Skein modules. Let $M$ be an oriented three-manifold and $\mathcal{L}$ the set of all isotopy classes of framed links in $M$. Let $\mathcal{R}=\mathbb{Z}\left[A^{ \pm 1}, d^{-1}\right]$, where $d=-A^{2}-A^{-2}$. Let $K(M)$ be the free $\mathcal{R}$-module generated by all elements of $\mathcal{L}$. We define the Kauffman bracket skein module of $M, \mathcal{K}(M)$, to be the quotient of $K(M)$ by the submodule generated by all expressions of the form

$$
\bigcirc \cup L-d L, \quad L-A L_{0}-A^{-1} L_{\infty},
$$

where $L, L_{0}$ and $L_{\infty}$ are three links which are identical except in a threeball where they are as in Figure 3. The Kauffman bracket skein module was computed for several manifolds. In particular, it was shown that there is an algebra structure on the skein module of $F \times I$, where $F$ is an oriented surface. The unit is the empty set and the product is given by including a copy of $F \times I$ into each of $F \times[0,1 / 2]$ and $F \times[1 / 2,1]$.

TheOREM $3.1([10,12])$. The skein algebra $\mathcal{K}\left(S^{1} \times I \times I\right)$ is isomorphic to the polynomial algebra $\mathcal{R}[z]$, where $z$ is represented by a curve in the annulus as in Figure 4.

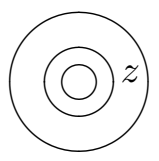

Fig. 4

Let $M$ be an oriented three-manifold and $\mathcal{G}$ the set of all isotopy classes of embeddings of ribbon graphs in $M$. Let $\mathcal{R G}$ be the free $\mathcal{R}$-module generated by $\mathcal{G}$. The Yamada skein module of $M$, which will be denoted by $\mathcal{Y}(M)$, is defined as the quotient of the module $\mathcal{R G}$ by the submodule generated by all expressions of the form:

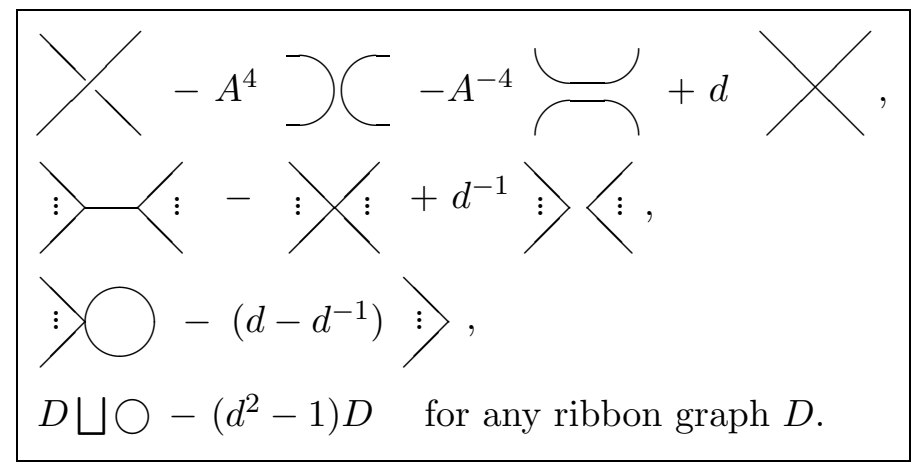

Fig. 5

One can define a graph skein theory for three-manifolds. This theory enjoys the same properties as the skein theory associated to links in three- 
manifolds (see [10]). The existence of the Yamada polynomial for spatial graphs is equivalent to the fact that $\mathcal{Y}\left(S^{3}\right)$ is isomorphic to $\mathcal{R}$. As in the case of the Kauffman bracket skein module there is an algebra structure on the Yamada skein module of $F \times I$, where $F$ is an oriented surface (see [3]).

THEOREM 3.2. The Yamada skein algebra of the solid torus $\mathcal{Y}\left(S^{1} \times I \times I\right)$ is isomorphic to the polynomial algebra $\mathcal{R}[z]$, where $z$ is represented by a curve in the annulus as in Figure 4.

Proof. In the annulus $S^{1} \times I$, we denote by $b_{n}$ the bouquet which is made up of $n$ non-trivial loops, with the convention that $b_{0}$ is the empty diagram $\emptyset$. Let $S_{n}$ be the graph with 2 vertices and $n+1$ edges as in Figure 6 . Finally, let $\theta_{n}$ be the $\theta_{n}$-curve (contained in some disk).
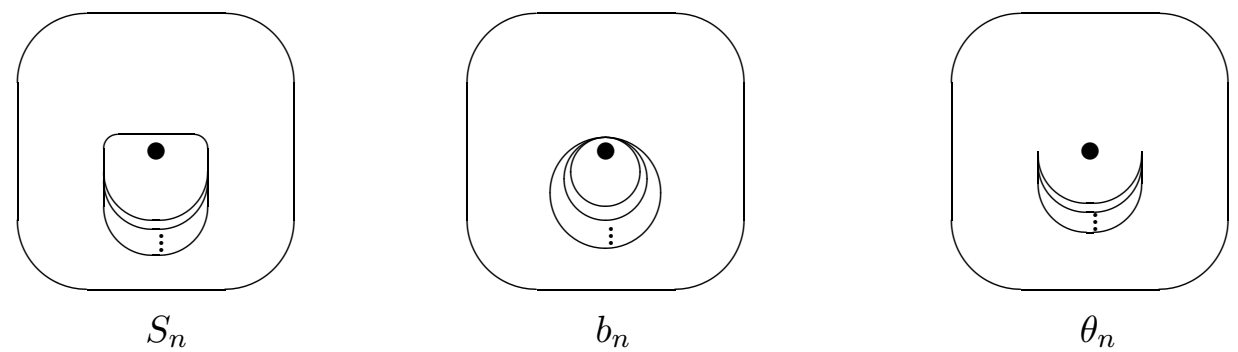

Fig. 6

Lemma 3.3. We have the following identities in $\mathcal{Y}\left(S^{1} \times I \times I\right)$ :

(i) $\theta_{n}=-d^{-1} \theta_{n-1}+\left(-\frac{d^{2}-1}{d}\right)^{n-2}\left(d^{2}-1\right) b_{0}$,

(ii) $S_{n}=-d^{-1} S_{n-1}+\left[\frac{-\left(d^{2}-1\right)}{d}\right]^{n-2} b_{1}$,

(iii) $b_{n}=S_{n}+d^{-1} \theta_{n}$.

Proof. A routine verification using the Yamada skein relations.

Let $D$ be a diagram of a spatial graph in the solid torus. It is easy to see that one can use the first Yamada skein relation to transform $D$ into a linear combination of diagrams with no crossings. By using the second Yamada skein relation, it is possible to transform each of those diagrams into a linear combination of diagrams such that each connected component has only one vertex. Finally, by applying the third and fourth Yamada skein relations, the diagram $D$ may be written as a linear combination of bouquets with no contractible cycles. This means that the set of all bouquets with no contractible cycles generates the Yamada skein module of the solid torus.

Now, according to (i) and (ii) of Lemma 3.3, each of $\theta_{n}$ and $S_{n}$ may be written as a linear combination of $b_{1}$ and $b_{0}$. Hence, (iii) implies that $b_{n}$ 
can be expressed as a linear combination of $b_{1}$ and $b_{0}$. Thus, we conclude that the Yamada skein module of the solid torus is generated by all links in the annulus $S^{1} \times I$, without trivial components but including the empty diagram. Finally, the skein algebra of the solid torus is isomorphic to the polynomial algebra $\mathcal{R}[z]$. This ends the proof of Theorem 3.2.

Throughout the rest of the paper we denote by $\tau_{2}$ the Temperley-Lieb algebra with the standard two generators

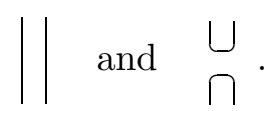

Let

$$
f_{1}=||-d^{-1} \stackrel{\cup}{\cap}
$$

be the Jones-Wenzl projector in $\tau_{2}$.

Let $G$ be a graph diagram. We define $G^{\prime}$ to be the linear combination of link diagrams obtained from $G$ by replacing each edge of $G$ by two planar strands with a projector $f_{1}$ in the cable, and by replacing each vertex of $G$ by a diagram as in Figure 7 (the figure illustrates the case of a four-valent vertex). Here, writing an integer $n$ beneath an edge $e$ means that this edge has to be replaced by $n$ parallel ones.
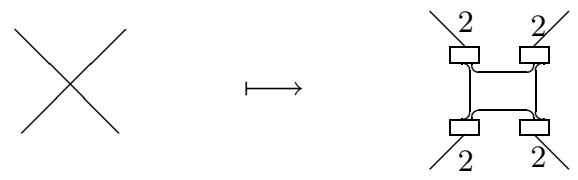

Fig. 7

Let $M$ be a three-manifold which is homeomorphic to the product $F \times I$, where $F$ is an oriented surface and $I$ is the unit interval. Let $\varphi$ be the map from $\mathcal{R G}$ to $\mathcal{K}(M)$ defined on the generators by $\varphi(G)=G^{\prime}$ and extended by linearity to $\mathcal{R G}$ (see also [14] and [15]).

Lemma 3.4. Let $\mathcal{Q}$ be the submodule of $\mathcal{R} \mathcal{G}$ generated by the Yamada skein relations. Then $\varphi(\mathcal{Q})=0$.

Proof. For the first skein relation we refer the reader to [5, p. 35]. Using the definition of $f_{1}$ and the fact that $f_{1}^{2}=f_{1}$, we can prove the result for the other skein relations. This is explained by the calculations below:

- the second skein relation: 

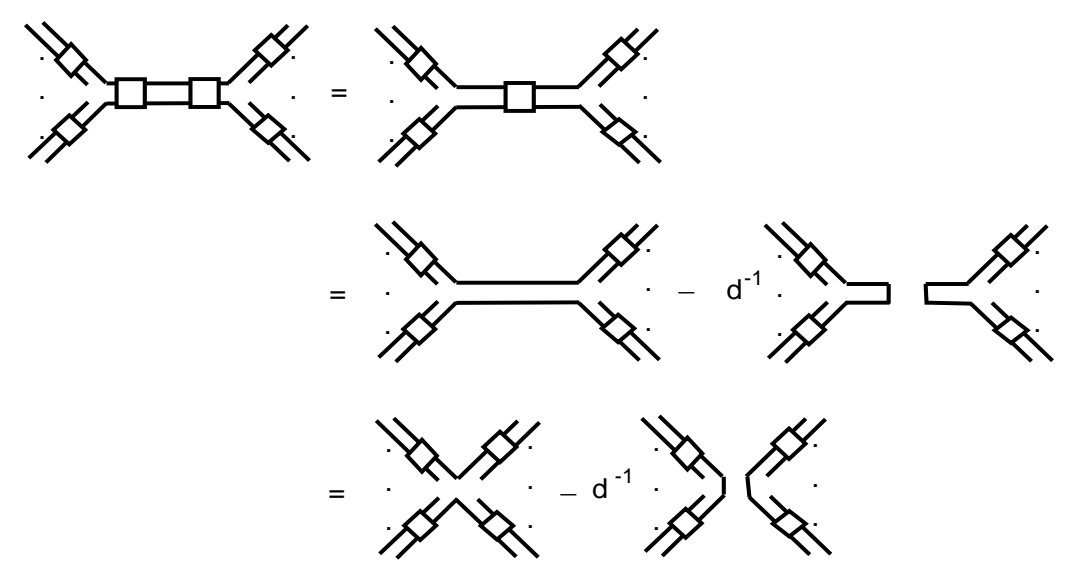

- the third skein relation:
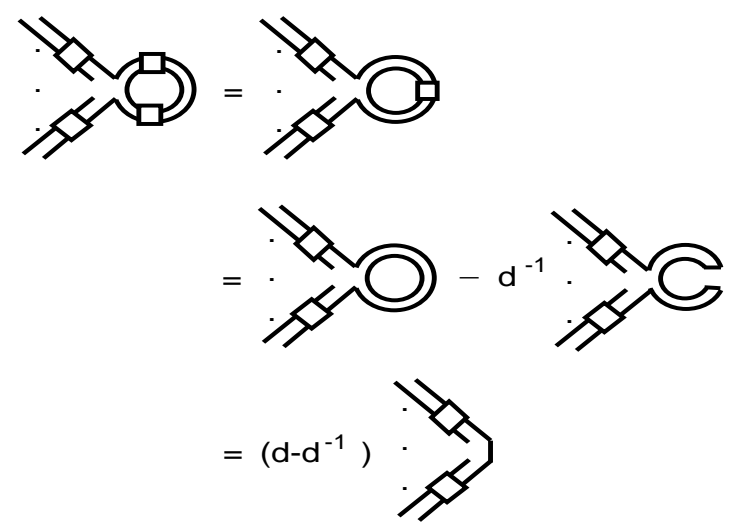

- the fourth skein relation:

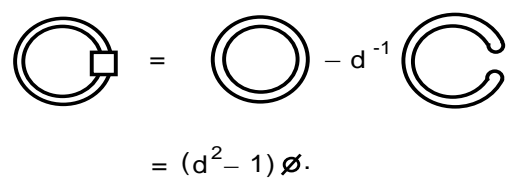

Since for every ribbon graph $D$ we have $\varphi(D \cup \bigcirc)=\varphi(D) \varphi(\bigcirc)$, the identity above implies that $\varphi\left(D \cup \bigcirc-\left(d^{2}-1\right) D\right)=0$. This ends the proof of Lemma 3.4 .

By Lemma 3.4, $\varphi$ defines a map from the Yamada skein module $\mathcal{Y}\left(S^{1} \times\right.$ $I \times I)$ to the Kauffman bracket skein module $\mathcal{K}\left(S^{1} \times I \times I\right)$; denote it by $\Phi$. It is easy to see that $\Phi$ is a homomorphism of algebras. Moreover, we have

LEMMA 3.5. The algebra homomorphism $\Phi$ is injective.

Proof. Direct computation shows that $\Phi(z)=z^{2}-1$. Hence, $\Phi$ is injective. 
4. Proof of Theorem 1.1. Let $\widetilde{G}$ be a $p$-periodic spatial graph. Let $L_{\widetilde{G}}$ be the set of all links that appear when we expand $\widetilde{G}$ as a linear combination of links using the map $\Phi$, that is,

$$
\Phi(\widetilde{G})=\sum_{D \in L_{\widetilde{G}}} d^{\alpha_{D}} D
$$

where $\alpha_{D}$ is an integer.

As there is an action of the finite cyclic group of order $p$ on the set of vertices and on the set of edges of $\widetilde{G}$, we can see easily that this group acts on $L_{\widetilde{G}}$. Moreover, there are two types of orbits:

- Orbits made up of only one link which is $p$-periodic. Let $L_{p, \widetilde{G}}$ be the subset of $L_{\widetilde{G}}$ made up of $p$-periodic links.

- Orbits made up of $p$ links which are cyclically permuted by the rotation.

In the second case the $p$ links are the same. Moreover, if $D$ and $D^{\prime}$ belong to the same orbit then $\alpha_{D}=\alpha_{D^{\prime}}$. Hence the contribution of the links from the same orbit adds to zero modulo $p$. Consequently, we have the following congruence modulo $p$ :

$$
\Phi(\widetilde{G}) \equiv \sum_{D \in L_{p, \widetilde{G}}} d^{\alpha_{D}} D
$$

If $D$ is $p$-periodic then by Theorem 2.2 , we have the following identity in the Kauffman bracket skein module:

$$
D \equiv \bar{D}^{p} \bmod p, d^{p}-d .
$$

Thus,

$$
\Phi(\widetilde{G}) \equiv \sum_{D \in L_{p, \widetilde{G}}} d^{\alpha_{D}} \bar{D}^{p} \bmod p, d^{p}-d .
$$

It is easy to see that if $D \in L_{p, \widetilde{G}}$ then $\alpha_{D}$ can be written $p \alpha_{D}^{\prime}$ for some integer $\alpha_{D}^{\prime}$. If we expand the graph diagram $\widetilde{G}$ into a linear combination of links using the map $\Phi$, we can see that the links that appear in the sum are exactly the quotients of the elements of $L_{p, \widetilde{G}}$. Moreover, if $D$ appears in $\Phi(\widetilde{G})$ with coefficient $\alpha_{D}$, then $\bar{D}$ appears in $\Phi(\underline{\widetilde{G}})$ with coefficient $\alpha_{D}^{\prime}$. This allows us to conclude that

$$
\Phi(\widetilde{G}) \equiv(\Phi(\widetilde{G}))^{p} \bmod p, d^{p}-d .
$$

Using the fact that $\Phi$ is an injective homomorphism of algebras we conclude that in the Yamada skein module of the solid torus we have

$$
\widetilde{G} \equiv(\widetilde{\widetilde{G}})^{p} \bmod p, d^{p}-d .
$$

This ends the proof of part (1) of Theorem 1.1.

Similar arguments are used to prove the second part. 
N. Chbili

\section{References}

[1] H. Bass and J. W. Morgan, The Smith Conjecture, Pure Appl. Math. 112, Academic Press, New York, 1994.

[2] N. Chbili, Les invariants $\theta_{p}$ des 3-variétés périodiques, Ann. Inst. Fourier (Grenoble) 51 (2001), 1135-1150.

[3] - , Graph-skein modules of three-manifolds and symmetry of spatial graphs, preprint.

[4] L. H. Kauffman, An invariant of regular isotopy, Topology 26 (1987), 395-407.

[5] L. H. Kauffman and S. L. Lins, Temperley-Lieb Recoupling Theory and Invariants of 3-Manifolds, Ann. of Math. Stud. 134, Princeton Univ. Press, 1994.

[6] W. B. R. Lickorish, The skein method for 3-manifold invariants, J. Knot Theory Ramif. 2 (1993), 171-194.

[7] Y. Marui, The Yamada polynomial of spatial graphs with $\mathbb{Z}_{n}$-symmetry, Kobe J. Math. 18 (2001), 23-49.

[8] K. Murasugi, The Jones polynomials of periodic links, Pacific J. Math. 131 (1988), 319-329.

[9] J. H. Przytycki, Skein modules of 3-manifolds, Bull. Polish Acad. Sci. Math. 39 (1991), 91-100.

[10] —, On Murasugi's and Traczyk's criteria for periodic links, Math. Ann. 283 (1989), 465-478.

[11] P. Traczyk, 10101 has no period 7: A criterion for periodicity of links, Proc. Amer. Math. Soc. 108 (1990), 845-846.

[12] V. G. Turaev, The Conway and Kauffman modules of the solid torus, Zap. Nauchn. Sem. LOMI 167 (1988),79-89 (in Russian); English transl.: J. Soviet Math. 52 (1990), 2799-2805.

[13] S. Yamada, An invariant of spatial graphs, J. Graph Theory 13 (1989), 537-551.

[14] - A topological invariant of spatial regular graphs, in: Knots 90, de Gruyter, 1992, 447-454.

[15] Y. Yokota, Topological invariants of graphs in 3-space, Topology 35 (1996), 77-87.

Department of Mathematics

Tokyo Institute of Technology

Oh-okayama Meguro Tokyo 152-8551, Japan

E-mail: chbili@math.titech.ac.jp

Received 23 February 2005;

in revised form 17 January 2006 